\section{Protéine cellulaire du prion : au-delà des encéphalopathies spongiformes}

Luc Dupuis, Jean-Philippe Loeffler
EA 3433

Laboratoire de signalisations

moléculaires et

neurodégénérescence,

Faculté de médecine,

4 , rue Blaise-Pascal,

67000 Strasbourg, France

loeffler@neurochem.

u-strasbg.fr

Quelle est la fonction de cette augmentation d'expression de $\mathrm{PrP}^{\mathrm{C}}$ dans ce contexte de neurodégénérescence? La réponse à cette question ne peut encore être définitive. En effet, la fonction cellulaire de $\operatorname{PrPC}$ reste encore largement débattue, bien que son extrême conservation évolutive atteste de son importance $(\rightarrow)$. Ainsi, les

$(\rightarrow) \mathrm{m} / \mathrm{s}$ 2002, $n^{\circ} 12$, p. 1267 ratives soient caractérisées par une augmentation générale des niveaux de $\mathrm{PrPC}^{C}[3]$. On observe ainsi un enrichissement en $\operatorname{Pr} P^{C}$ dans les plaques séniles de patients atteints de maladie d'Alzheimer [4], ainsi que dans les neurites dystrophiques de ces mêmes patients, ces deux types de structure étant caractéristiques de la maladie d'Alzheimer. Cette observation semble relativement spécifique, aucune immunoréactivité de $\operatorname{PrP} \mathrm{P}^{\mathrm{C}}$ 'étant retrouvée dans d'autres types d'inclusions cellulaires comme les corps de Lewy ou de Bunina. souris invalidées pour $\operatorname{PrP}{ }^{C}$ ne présentent pas de phénotype majeur [1]. PrPC est une protéine essentiellement exprimée dans le système nerveux central et particulièrement au niveau des terminaisons synaptiques [5]. II semble acquis qu'elle lie le cuivre [6], ce qui lui conférerait une activité antioxydante ou un rôle dans les mouvements cellulaires du cuivre [7]. Par ailleurs, un rôle lui a été attribué dans le contrôle de la survie neuronale, peut-être par l'activation de voies de transduction neuroprotectrices [8-10]. D'autres auteurs montrent que $\mathrm{PrPC}$ pourrait dans certaines conditions être au contraire proapoptotique [11]. Quelle que soit la fonction exacte de PrPC, l'augmentation de son expression au cours des maladies neurodégénératives apparaît comme une réponse physiologique (neuroprotectrice?) possible à une situation de stress cellulaire.

Parmi l'ensemble des pathologies neurodégénératives étudiées, la sclérose latérale amyotrophique (SLA) fait exception en présentant une régulation de l'expression de PrPC opposée aux autres maladies neurodégénératives. Deux études indépendantes arrivent en effet à cette conclusion. Notre équipe a montré que les

Tableau I. Pathologies impliquant la protéine PrPC. 
niveaux d'expression de $\operatorname{PrP}^{C}$ sont diminués, tant en ce qui concerne l'ARNm que la protéine, dans un modèle de souris porteuses d'une mutation de la SOD (superoxyde dismutase-1) identique à celle retrouvée dans certaines formes de SLA. Cette réduction de $\operatorname{Pr} P^{C}$ ne s'accompagne pas d'une accumulation de PrP résistante aux protéases. Elle est spécifique de la mutation SOD1, car elle n'est pas retrouvée chez des souris surexprimant la forme sauvage de la SOD1 [12]. Enfin, la répression de $\operatorname{Pr} P^{C}$ n'est pas dépendante de phénomènes de dénervation: en effet, elle n'est pas retrouvée dans la moelle lombaire de souris dont le nerf sciatique a été sectionné [13]. L'équipe de Budka a récemment confirmé nos observations réalisées chez la souris auprès de patients atteints de SLA [3] : il existe, par rapport à des témoins, une diminution significative des niveaux de $\operatorname{PrP}^{C}$ dans 9 cas. La SLA est donc un cas particulier parmi les maladies neurodégénératives.

La diminution d'expression de PrPC contribue-t-elle à la neurodégénérescence au cours de la SLA ? Une manière de répondre à cette question serait de croiser des modèles animaux de SLA porteurs de mutations SODl avec des animaux issus de lignées transgéniques exprimant des niveaux variés de $\operatorname{PrPC}$. Ces études devraient permettre de déterminer directement si la protéine $\mathrm{Pr}^{\complement} \mathrm{C}^{\mathrm{C}}$ possède un rôle neuroprotecteur in vivo. L'observation inattendue d'une régulation négative de $\operatorname{Pr} P^{C}$ dans la SLA suggère que la protéine $\operatorname{PrP}^{C}$ est susceptible de jouer un rôle dans la physiopathologie de maladies neurodégénératives autres que les formes génétiques ou transmissibles des maladies à prion. L'expression de cette protéine n'est cependant pas restreinte au système nerveux central; la protéine $\operatorname{Pr} P^{C}$ est retrouvée dans de nombreux tissus, et en particulier dans le muscle squelettique.

Des études récentes suggèrent d'ailleurs que cette protéine $\operatorname{Pr} P^{C}$ pourrait également jouer un rôle dans différentes maladies neuromusculaires. Ainsi, les niveaux de $\mathrm{PrPC}^{\mathrm{C}}$ sont nettement augmentés dans certains cas sporadiques de myosites à inclusion, de polymyosites et de dénervations neurogènes [14, 15]. Ces résultats sont parfaitement cohérents avec nos propres données montrant que l'expression de $\mathrm{PrPC}$ est nettement augmentée dans le muscle gastrocnémien de souris après section du nerf sciatique, montrant que le gène codant pour la $\operatorname{Pr} \mathrm{P}^{\mathrm{C}}$ est un gène de réponse à la dénervation. Une telle augmentation de $\operatorname{PrP} P^{C}$ dans le tissu musculaire est susceptible d'être délétère: en effet, les premières lignées de souris transgéniques surexprimant PrPC présentaient un phénotype neuromusculaire très accentué, caractérisé notamment par une nécrose du tissu musculaire et une neuropathie démyélinisante [16]. De plus, la surexpression d'un mutant insertionnel de $\operatorname{Pr} P^{C}$ associé à des formes familiales d'ESST produit une myopathie primaire [17], montrant ainsi que les fonctions potentielles de $\mathrm{PrPC}^{\mathrm{C}}$ ne sont pas restreintes au système nerveux central.

La protéine $\operatorname{PrPC}$, initialement reconnue comme un déterminant des maladies à prion, est donc vraisemblablement beaucoup plus que le vecteur ou le support endogène de ces pathologies. Elle semble aussi être un acteur majeur de voies de transduction potentiellement importantes dans la survie neuronale, ce qui explique son implication générale dans de nombreuses pathologies neurodégénératives, mais aussi musculaires. II reste cependant à élucider quelles pourraient être la (ou les) fonction(s) cellulaire(s) contrôlée (s) par PrPC. Parmi celles-ci, on peut évoquer une fonction mitochondriale. La contribution de la mitochondrie aux pathologies neurodégénératives est maintenant bien établie dans les maladies d'Azheimer et de Parkinson, ainsi que dans la SLA, et il est intéressant de noter que l'un des principaux effets de l'invalidation du gène de la $\operatorname{PrP}^{C}$ est une altération du nombre et de la morphologie des mitochondries [18]. La protéine $\operatorname{Pr} P^{C}$ pourrait donc être l'un des chaînons manquants de la régulation extracellulaire de la biogenèse et du fonctionnement mitochondrial. On comprend dès lors les conséquences d'une modification même minime de sa structure ou de son expression. $\diamond$

Cellular prion protein: more than what beyond spongiform encephalopathies

\section{REMERCIEMENTS}

Les auteurs remercient le GIS pour son soutien financier.

\section{RéFÉRENCES}

1. Bueler $\mathrm{H}$, Fischer M, Lang $Y$, et al. Normal development and behaviour of mice lacking the neuronal cellsurface PrP protein. Nature 1992; 356: 577-82.

2. Prusiner SB, Scott MR, De Armond SJ, Cohen FE. Prion protein biology. Cell 1998; 93: 337-48.

3. Kovacs G, Zerbi P, Voigtlander T, et al. The prion protein in human neurodegenerative disorders. Neurosci Lett 2002; 329: 269.

4. Ferrer I, Blanco R, Carmona $M$, et al. Prion protein expression in senile plaques in Alzheimer's disease. Acta Neuropathol (Berl) 2001; 101: 49-56.

5. Herms J, Tings T, Gall S, et al. Evidence of presynaptic location and function of the prion protein. J Neurosci 1999; 19: 8866-75.

6. Brown DR, Qin K, Herms JW, et al. The cellular prion protein binds copper in vivo. Nature 1997; 390: 684-7.

7. Brown DR. Prion and prejudice: normal protein and the synapse. Trends Neurosci 2001; 24 : 85-90.

8. Mouillet-Richard $S$, Ermonval M, Chebassier C, et al. Signal transduction through prion protein. Science 2000; 289: 1925-8.

9. Kuwahara C, Takeuchi AM, Nishimura T, et al. Prions prevent neuronal cell-line death. Nature 1999; 400: 225-6. 
10. Bounhar $y$, Zhang $y$, Goodyer CG, LeBlanc A. Prion protein protects human neurons against Bax-mediated apoptosis. J Biol Chem 2001 . 276: 39145-9.

11. Paitel $\varepsilon$, Fahraeus R, Checler F. Cellular prion protein sensitizes neurons to apoptotic stimuli through Mdm2-regulated and P53-dependent caspase 3-like activation. J Biol Chem 2003; 278: 10061-6.

12. Dupuis L, Mbebi C, Gonzalez de Aguilar JL, et al. Loss of prion protein in a transgenic model of amyotrophic lateral sclerosis. Mol Cell Neurosci 2002; 19: 216-24.
13. Dupuis L, Di Scala F, Gonzalez de Aguilar JL, Rene F, De Tapia M, LoefflerJP. Denervation is not the primary cause of prion protein downregulation occuring in the spinal cord of a transgenic model of amyotrophic lateral sclerosis. Ann Ny Acad Sci 2002; 973: 116-9.

14. Zanusso G, Vattemi G, Ferrari $S$, et al. Increased expression of the normal cellular isoform of prion protein in inclusion-body myositis, inflammatory myopathies and denervation atrophy. Brain Pathol 2001; 11: 182-9.
15. Lampe JB, Walter MC, Reichmann $\mathrm{H}$. Neurodegenerationassociated proteins and inflammation in sporadic inclusion-body myositis. Adv Exp Med Biol 2001; 487: 219-28.

16. Westaway D, DeArmond SJ, Cayetano-Canlas J, et al. Degeneration of skeletal muscle, peripheral nerves, and the central nervous system in transgenic mice overexpressing wild-type prion proteins. Cell 1994; 76: 117-29.
17. Chiesa R, Pestronk A, Schmidt RE, et al. Primary myopathy and accumulation of PrPSc-like molecules in peripheral tissues of transgenic mice expressing a prion protein insertional mutation. Neurobiol Dis 2001; 8: 279-88.

18. Miele $G$, Jeffrey $M$, TurnbullD, Manson J, Clinton, M. Ablation of cellular prion protein expression affects mitochondrial numbers and morphology. Biochem Biophys Res Commun 2002; 291: 372-7.

\section{NOUVELLE}

\section{Équations de la soudure épithéliale}

Raphaël Rousset, Luis Almeida, Stéphane Noselli

> La réparation tissulaire, en particulier la fermeture des plaies, est un acte réflexe des tissus permettant d'assurer leur continuité et la défense face aux infections [1]. L'imperfection notoire de la réparation se manifeste par l'apparition de cicatrices d'aspect peu esthétique. De multiples stratégies, le plus souvent empiriques, ont été élaborées au fil du temps pour tendre vers la cicatrisation parfaite (c'est-à-dire rapide et sans trace), mais le remède miracle reste à découvrir. Une condition importante pour l'élaboration de traitements efficaces est le développement de modèles animaux offrant un large éventail d'approches et de méthodologies. L'un de ces modèles est la fermeture dorsale (FD) de l'embryon de drosophile. La FD représente une étape clé de l'embryogenèse au cours de laquelle la région dorsale de l'embryon, occupée par l'amnioséreuse, est recouverte par deux feuillets épithéliaux migrant l'un vers l'autre (Figure 1A) [2, 3]. En l'espace de 2 heures, ils se rejoignent au niveau de la ligne dorsale et fusionnent parfaitement. Vu de dessus, le rapprochement des deux marges de l'ectoderme en cours de migration peut être comparé au clignement d'un œil dont les coins (canthus) se fermeraient avant le centre selon un processus de type «fermeture éclair» (zipping). De façon intéressante, ce processus naturel de soudure épithéliale peut être assimilé à une cicatrisation programmée au cours du développement $[4,5]$.

L'étude génétique, moléculaire et cellulaire de la FD a permis d'identifier trois structures essentielles à ce processus: l'amnioséreuse, l'ectoderme latéral et, délimitant ces deux tissus, la marge de
R. Rousset, S. Noselli : Institut de Recherches Signalisation, Biologie du Développement et Cancer, Cnrs UMR 6543, Université de Nice-Sophia Antipolis,

Centre de Biochimie, Parc

Valrose, 06108 Nice Cedex 2, France.

L. Almeida: Laboratoire J. A. Dieudonné, Cnrs UMR 6621, Parc Valrose, 06108 Nice

Cedex 2, France. noselli@unice.fr

l'ectoderme en migration (Figure IA) [2, 3]. Celle-ci est notamment caractérisée par la présence d'un câble riche en actine qui relie chaque cellule [6], ainsi que d'extensions du cytosquelette (filopodes, lamellipodes) importantes pour la suture des marges [7]. Cependant, la contribution relative de ces trois structures au mouvement coordonné de la FD est totalement inconnue. Afin de mieux comprendre les forces et tensions mises 